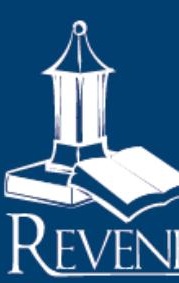

\title{
EVENTOS DE PRECIPITACIÓN PLUVIAL ASOCIADOS A DIEZ ENFERMEDADES DE DECLARACIÓN OBLIGATORIA EN LA ZONA DE PEREZ ZELEDÓN ${ }^{1}$
}

Consuelo Cubero Alpízar²

Institución: Universidad de Costa Rica

\section{COMO CITAR}

Cubero, E. (2012) Eventos de precipitación pluvial asociados a diez enfermedades de declaración obligatoria en la zona de Pérez Zeledón[En línea].Rev. Actual en Costa Rica, 22, 1-10 [citado (fecha)]. Disponible World World Wide Web: <http://www.revenf.ucr.ac.cr/eventos.pdf> ISSN $1409-4568$

\section{RESUMEN}

El presente artículo muestra los resultados sobre el comportamiento de 10 problemas de salud de reporte obligatorio y su relación con la precipitación pluvial en el cantón de Pérez Zeledón durante el periodo 2005 al 2010. Es una investigación documental que utilizó como fuentes de información las estadísticas de enfermedades de reporte obligatorio proporcionadas por la Caja Costarricense de Seguro Social (CCSS), además de datos sobre precipitación procedentes de dos estaciones meteorológicas proporcionados por el Instituto Costarricense de Electricidad (ICE). Se elaboró una matriz de datos para el procesamiento y análisis de la información. Como resultado se encontró que con el aumento en la precipitación pluvial aumenta la incidencia de las enfermedades estudiadas; se determinó que algunas tienen un comportamiento muy similar; se observó que tanto las enfermedades como la precipitación pluvial coinciden en los picos de mayor incidencia. Se concluye que el aumento de la precipitación pluvial constituye un factor importante en el aumento en la incidencia de muchos problemas de salud, lo cual apela a las instancias de salud para que las atiendan e implementen estrategias que disminuyan el riesgo de que la población padezca estas enfermedades.

PALABRAS CLAVE: salud-publica, gestión-de-riesgo, enfermedades-infectocontagiosas, precipitación

\footnotetext{
${ }^{1}$ Fecha de recepción: 13 de noviembre del 2011

Fecha de aceptación: 5 de marzo de 2012

2 Enfermera. Docente. Escuela de Enfermería de la Universidad de Costa Rica. Magister en Gestión de riesgo en desastres y atención de emergencias Correo electrónico: ccubero.alpizar@gmail.com
} 


\title{
RAINFALL EVENTS ASSOCIATED WITH TEN DISEASES OF DECLARATION REQUIRED IN THE AREA OF PEREZ ZELEDON ${ }^{1}$
}

Consuelo Cubero Alpízar²

INSTITUTION: University of Costa Rica

CITED

Cubero, E. (2012) Eventos de precipitación pluvial asociados a diez enfermedades de declaración obligatoria en la zona de Pérez Zeledón [En línea].Rev. Actual en Costa Rica, 22, 1-10 [cited (Date)].Disponible World World Wide Web: <http://www.revenf.ucr.ac.cr/eventos.pdf> ISSN 14094568

\begin{abstract}
This article presents results on the performance of 10 health problems of obligatory report and its relation to rainfall in the county of Perez Zeledon during the period 2005 to 2010. It is a documental research in which were used as sources of information and statistics about diseases of obligatory report provided by the Social Security Fund (CCSS), precipitation data from two weather stations provided by the Instituto Costarricense de Electricidad (ICE .) A matrix of data processing and data analysis. As a result it was found that with the increase in rainfall increases the incidence of the diseases studied, we found that some have a very similar behavior, showing that both conditions such as rainfall peaks coincide in the highest incidence. We conclude that increased rainfall is an important factor in the increased incidence of many health problems, leading to instances of timely health care for its implementation of strategies to decrease the risk in the population of developing these diseases.
\end{abstract}

Keywords: public-health, management, risk-infectious-diseases, precipitation

\footnotetext{
${ }^{1}$ Date of reception : 13 de noviembre del 2011

Date of acceptance: 5 de marzo del 2012

${ }^{2}$ Enfermera. Docente. Escuela de Enfermería de la Universidad de Costa Rica. Magister en Gestión de riesgo en desastres y atención de emergencias Correo electrónico: ccubero.alpizar@gmail.com
} 


\section{INTRODUCCIÓN}

La salud de las comunidades se ve afectada por una serie de factores ambientales, entre los que se encuentran la precipitación pluvial, la cual requiere sistemas de vigilancia que brinden medidas para disminuir el riesgo de padecer enfermedades que se pueden expandir rápidamente en la población. Al respecto, la Epidemiología es la disciplina que se encarga de estudiar este tipo de eventos que afectan a la población. Puede definirse de la siguiente manera:

La Epidemiología como disciplina sociomédica tiene como objeto de estudio básico la situación de salud de las poblaciones. El predominio de un enfoque poblacional refuerza el papel de las intervenciones de carácter preventivo y de promoción sobre colectividades. Una de sus áreas fundamentales de trabajo es la vigilancia en salud, herramienta esencial para la toma de decisiones en salud pública." (Fariñas, Coutin y Rodríguez, 2009)

Partiendo de lo anterior, se puede afirmar que la investigación sobre factores ambientales es una temática de la que se ocupa el enfoque poblacional, debido a que incide en la salud de las personas; ejemplo de ello son los cambios en el clima, especialmente los asociados a la estación lluviosa, los cuales ofrecen un ambiente propicio para la proliferación de vectores, así como para la multiplicación y transmisión de virus y bacterias causantes de enfermedades.

El efecto que estos eventos tienen sobre la salud de las personas es un tema poco explorado, como también lo es la distribución del comportamiento a lo largo del año en Costa Rica.

En relación con el comportamiento de los eventos causantes de emergencias y desastres en el territorio nacional se encuentra que

El mayor número de tipos de eventos que han provocado daños importantes a lo largo del período 1988-2009, lo conforman los hidrometeorológicos con 34 eventos (82,9\%), entre los cuales 32 corresponden a exceso de precipitación y 2 a déficit de precipitación o sequía. Los sismos con potencial destructor ocurridos durante el período de estudio son cinco y representan el 12,2\% de los eventos del período. (Flores, Salas, Astorga y Rivera, 2010, p.7)

Fallas y Valverde (2007) refieren que los eventos hidrometeorológicos extremos son parte del comportamiento normal del clima, pero que en los últimos años se les ha conferido mayor importancia por el impacto que están teniendo en el desarrollo y los medios de producción.

Respecto al impacto económico de los fenómenos en mención, Flores, R., Salas, J., Astorga, M., Rivera, J. (2010) mencionan que "el exceso de precipitación provocó pérdidas por 1.053 millones de dólares, 
equivalentes al 57,8 \% del total" (p.7), sin embargo, su efecto en la salud de las personas por las enfermedades infectocontagiosas asociadas al contacto con material contaminado, así como las pérdidas en la infraestructura de las instituciones, es un tema completamente invisibilizado.

Por otro lado, las estadísticas de los últimos 40 años muestran que las inundaciones son las mayores causantes de emergencias y desastres. En Costa Rica, hubo un $51 \%$ del total de eventos registrados, siendo San José la provincia de que reúne un $28 \%$ del total de esos eventos. Otros investigadores, como la Blaikie, Cannon, Davis, y Wisner (1996); Ahern, Kovats, Wilkinson, Few, \& Matthies (2005); Fawtrell \& Kay (2008), hacen hincapié en el daño que el aumento en la precipitación y las inundaciones provoca a la salud.

Además, otras publicaciones más recientes como la de Giorgadze, Maisuradze, Japaridze, Utiashvili y Abesadze (2011) recalcan el efecto que los eventos de origen natural tienen sobre la salud de las personas al mencionar que

Cada año millones de personas están afectadas por los desastres naturales y provocados por el hombre en todo el mundo. Tornados, huracanes, lluvias torrenciales y terremotos dio lugar a decenas de miles de muertos y muchos más afectada. De hecho, los desastres no sería un desastre si no fuera por su efecto sobre la población humana. Los vínculos entre el medio natural y la salud humana se han sugerido desde hace siglos. Los desastres de la historia han tenido un impacto significativo sobre el número, estado de salud y estilo de vida de las poblaciones. Se induce: Las muertes, lesiones graves, que requieren tratamientos extensos, mayor riesgo de enfermedades transmisibles, daños a las instalaciones de salud, daños a los sistemas de agua, escasez de alimentos, los movimientos de población. Los autores se centraron en los desastres naturales, causados por fuerzas naturales y no por los actos relacionados con el comportamiento humano y que afectan a una gran población en una región geográfica amplia. Describir los efectos generales de los desastres sobre la salud, que no pretende cubrir todas las contingencias. Revisión de la literatura reciente sobre las emergencias humanitarias se ha demostrado que las consecuencias de salud pública de los desastres naturales son complejos. Los desastres afectan directamente la salud de la población que resulta en un trauma físico, enfermedad aguda y trauma emocional. Además, los desastres pueden aumentar la morbilidad y la mortalidad asociada con la enfermedad crónica y las enfermedades infecciosas a través del impacto sobre el sistema de salud. (s.p.)

Ante esta panorámica, los sistemas de vigilancia epidemiológica cumplen un papel indiscutible, independiente del nivel en el que se implemente, por ejemplo

En la atención primaria, la vigilancia es un componente de trabajo para realizar el análisis permanente de la situación de salud de las poblaciones, lo que le permite identificar los problemas principales en conjunto con todos los actores sociales y trazar un plan de acción 
correctivo. Dentro del nivel secundario, la vigilancia es una aliada insustituible en el reconocimiento de los principales problemas de salud de los territorios a los que les brindan servicios. Es, además, un medio de detección de las desviaciones de las acciones hospitalarias que deben ser corregidas para alcanzar una mayor calidad en la atención prestada. En el nivel terciario acompaña a todo el proceso estratégico de toma de decisiones mediante la elaboración de pronósticos y la utilización de técnicas rápidas de vigilancia.(Fariñas, Coutin y Rodríguez, 2009 s.p.)

La vigilancia de la salud debe trascender la simple recolección de datos, pues es necesario tomar decisiones ante el aumento de ciertos problemas de salud, tal es el caso de los eventos hidrometeorológicos, especialmente el aumento en la precipitación pluvial.

Gracias a la vigilancia epidemiológica se ha podido identificar el efecto que las precipitación pluvial tiene en la incidencia de casos de dengue, accidentes ofídicos, influenza, infecciones respiratorias agudas superiores (IRAS), Leptospirosis, Leishmaniasis, diarreas, salmonelosis, shigellosis y tosferina. Dichas enfermedades por su forma de transmisión pueden estar asociadas con el aumento en la precipitación pluvial y también con las inundaciones, lo cual muchas veces pasa desapercibido y el costo de su atención permanece invisible, de manera que pierde su vínculo con la gestión del riesgo ante desastres.

Ante la panorámica mencionada anteriormente y la escasa investigación existente en la país sobre el tema, se planteó como objetivo analizar la precipitación pluvial asociada a la presencia de diez enfermedades de declaración obligatoria en el cantón de Pérez Zeledón durante el quinquenio 2005-2010.

\section{MATERIALES Y MÉTODOS}

La investigación se realizó en el cantón de Pérez Zeledón, número 19 de la provincia de San José (Costa Rica), ubicado en la Región Brunca (sur del país). Su cabecera y principal ciudad es San Isidro de El General, la cual cuenta con una población aproximada de 135429 habitantes siendo uno de los cantones más poblados, según datos del Instituto Nacional de Estadísticas y Censos (INEC, 2011)

El estudio fue de tipo documental en el cual se utilizaron datos sobre la precipitación pluvial acumulada por mes, suministrados por el Instituto Costarricense de Electricidad (ICE) (estaciones meteorológicas "Linda" y "Alto San Juan", respectivamente).En cuanto a la información referente a emergencias o desastres en Costa Rica, se usó como fuente la base de datos DesInventar (2011), la cual compila todos los reportes suministrados por la Comisión Nacional de Gestión de Riesgos y Atención de Emergencias (CNE). Además, se recopilaron datos sobre el comportamiento de diez enfermedades de declaración obligatoria en la región estudiada, información proporcionada por la Caja Costarricense de Seguro Social (CCSS). El periodo analizado correspondió al quinquenio 2005-2010.

Los criterios de inclusión para estas enfermedades son los siguientes: 
- De tipo infectocontagiosas o sensibles a cambios ambientales.

- Estar incluidas dentro del reporte de enfermedades de declaración obligatoria.

- Ser ocasionadas por alteraciones del ambiente asociados a los eventos de inundación.

- Contar con datos para el periodo en estudio.

De esta manera, las enfermedades que cumplieron con los criterios fueron: dengue, influenza, diarrea, shigellosis, salmonelosis, influenza, IRAS, Tosferina, Leptospirosis, leishmaniasis.

La información de precipitación pluvial, fue agrupada en una matriz de datos por mes y año. De igual forma, se trataron los datos sobre las diez enfermedades de reporte obligatorio, con el fin de hacer una comparación y análisis de ambos eventos.

\section{Consideraciones éticas}

Dentro de los aspectos éticos del trabajo se encuentran el respeto al principio de privacidad, por tanto, se mantiene en el anonimato las personas reportadas que padecieron alguna de las enfermedades analizadas. Además, se respetó el principio de veracidad al hacer uso de la información suministrada por las diferentes instituciones, así como al analizar tales datos. Además, se solicitaron los permisos a las instituciones responsables para la utilización de sus respectivas bases de datos.

\section{RESULTADOS}

A continuación, se presentan los resultados según cada uno de los problemas de salud, estudiados para el quinquenio 2005-2010. Accidentes ofidios: obsérvese la mayor incidencia mensual en los siguientes datos:

\begin{tabular}{|c|c|c|}
\hline 1. $\quad$ Año/Mes & No. de casos & Precipitación pluvial (mm) \\
\hline \multicolumn{3}{|l|}{2005} \\
\hline Agosto & 9 & 382,6 \\
\hline Setiembre & 15 & 795,1 \\
\hline Octubre & 9 & 553,9 \\
\hline \multicolumn{3}{|l|}{2006} \\
\hline Mayo & 10 & 406,05 \\
\hline Junio & 13 & 362,1 \\
\hline Agosto & 10 & 405,85 \\
\hline \multicolumn{3}{|l|}{2007} \\
\hline Agosto & 7 & 506,25 \\
\hline Setiembre & 7 & 609,3 \\
\hline \multicolumn{3}{|l|}{2008} \\
\hline Agosto & 5 & 577,75 \\
\hline Octubre & 4 & 615,95 \\
\hline
\end{tabular}


Durante el 2009, el comportamiento es desigual entre ambos fenómenos y para el 2010 se destaca en primer lugar una marcada disminución en la incidencia respecto de los años anteriores; además, la mayor cantidad de accidentes se presentan en el mes de enero ( 6 casos y 05,25 precipitación pluvial) cuando se reporta la menor precipitación. A pesar de lo anterior, aparecen comportamientos similares entre el aumento en la precipitación y los accidentes ofídicos para los meses de junio, agosto y noviembre.

Otro aspecto interesante que se desprende es que la incidencia anual de accidentes ofídicos ha ido disminuyendo; por ejemplo, en el 2005 se presentaron 69 casos; 97 casos durante el 2006; 45, en el 2007, 42 en el 2008; 32, en el 2009 y 14 en el 2010. Para este último año se destaca que la precipitación fue más alta que los años anteriores.

2. Dengue: obsérvese los siguientes datos en cuanto al número de casos presentados y su relación con la precipitación pluvial:

\begin{tabular}{c|c|c|c|c|c|c|c|c|c|c}
\hline \multirow{2}{*}{ Año } & \multicolumn{2}{|c|}{ Mayo } & \multicolumn{2}{c|}{ Junio } & \multicolumn{2}{c|}{ Julio } & \multicolumn{2}{c|}{ Agosto } & \multicolumn{2}{c}{ Setiembre } \\
\cline { 2 - 11 } & casos & $\begin{array}{c}\text { Prec. pluvial } \\
(\mathrm{mm})\end{array}$ & casos & $\begin{array}{c}\text { Prec. pluvial } \\
(\mathrm{mm})\end{array}$ & casos & $\begin{array}{c}\text { Prec. } \\
\text { pluvial } \\
(\mathrm{mm})\end{array}$ & casos & $\begin{array}{c}\text { Prec. pluvial } \\
(\mathrm{mm})\end{array}$ & casos & $\begin{array}{c}\text { Prec. pluvial } \\
(\mathrm{mm})\end{array}$ \\
\hline 2005 & - & - & 381 & 331 & - & - & 278 & 382,6 & 160 & 795,1 \\
\hline 2006 & 26 & 406,05 & 72 & 362,1 & 75 & 317,1 & - & - & - & - \\
\hline 2007 & 26 & 310,55 & 24 & 425,8 & 50 & 284,45 & - & - & - & - \\
\hline 2008 & 19 & 458,4 & 59 & 325,5 & 60 & 422,05 & - & - & - & - \\
\hline
\end{tabular}

En el año 2009, se presentó un comportamiento similar entre ambos fenómenos; no obstante, es el año que tiene la menor precipitación pluvial y la menor incidencia de casos de dengue, al punto de que se reportó un aumento de incidencia en los meses de setiembre y octubre ( 247 casos). En el año 2010, el dato más significativo se encontró en junio con 239 casos de dengue y una precipitación pluvial de 409,1mm.

3. Diarreas: se observó que este problema de salud tiene un comportamiento poco ligado con el aumento en la precipitación pluvial, ya que muestra aumentos durante la estación seca, de igual forma para los meses de la estación lluviosa para todo el quinquenio. Obsérvese el siguiente dato según año y casos.

\begin{tabular}{c|c}
\hline Año & No. de casos \\
\hline 2005 & 4982 \\
\hline 2006 & 4463 \\
\hline 2007 & 5944 \\
\hline 2008 & 4311 \\
\hline $2009 *$ & 3957 \\
\hline 2010 & 4644 \\
\hline
\end{tabular}

* Año que tiene menor precipitación y menor incidencia de casos 
4. Salmonellosis: se observó que durante el año 2005 aumentó la incidencia de esta enfermedad en abril (3 casos/precipitación pluvial 149,7 mm), junio(2 casos/precipitación pluvial $331 \mathrm{~mm}$ ), y agosto(2 casos/precipitación pluvial de 382,6 mm). En el 2006, se reportó un caso por mes, en donde se destaca la precipitación pluvial en marzo $(54,95 \mathrm{~mm})$, abril $(190,8 \mathrm{~mm})$, mayo $(406,05 \mathrm{~mm})$ y julio $(317,1 \mathrm{~mm})$. En el 2007, hubo un aumento en mayo ( 4 casos/ precipitación pluvial de $310,55 \mathrm{~mm}$ ) y junio ( 5 casos/precipitación pluvial de 425,8mm), situación que se repite durante el 2008 en octubre ( 2 casos/precipitación pluvial de $615,95 \mathrm{~mm})$. Durante los años 2009 y 2010 no hubo reportes de esta enfermedad.

5. Shigellosis: respecto de esta enfermedad, se presentan los siguientes datos: para el año 2005, se presentaron 3 casos en abril (precipitación pluvial 149,7 mm), seguido de 8 casos en junio (precipitación pluvial 331,0 mm), 16 casos en agosto (precipitación pluvial 382,6 mm) y 4 casos en setiembre (precipitación 795,1 mm). En el 2007, se destacó un registro de 5 casos en junio (con una precipitación pluvial de 425,8 mm) y 3 casos en setiembre (precipitación pluvial 609,3 mm) En el 2006, este comportamiento fue menos marcado y se presentó principalmente en mayo ( 2 casos/precipitación pluvial de 406,05 mm) y julio(2 casos/precipitación pluvial de 317,1 mm). En el 2007, se presentó un repunte en la incidencia de esta enfermedad principalmente en mayo (12 casos y una precipitación pluvial de 310,55)En el 2008, el comportamiento es más puntual, lo fenómeno observado en mayo(3 casos/precipitación pluvial de $458,4 \mathrm{~mm}$ ) y octubre ( 2 casos/precipitación pluvial de 615,95mm). En el 2009 y 2010 la incidencia es muy baja y no existe relación aparente entre ambos fenómenos.

6. Influenza: presentó el siguiente comportamiento:

\begin{tabular}{c|c|c|c|c|c|c|c|c|c|c}
\hline \multirow{2}{*}{ Año } & \multicolumn{2}{|c|}{ Mayo } & \multicolumn{2}{c|}{ Junio } & \multicolumn{2}{c|}{ Julio } & \multicolumn{2}{c|}{ Agosto } & \multicolumn{2}{c}{ Setiembre } \\
\cline { 2 - 11 } & casos & $\begin{array}{c}\text { Prec. pluvial } \\
(\mathrm{mm})\end{array}$ & casos & $\begin{array}{c}\text { Prec. pluvial } \\
(\mathrm{mm})\end{array}$ & casos & $\begin{array}{c}\text { Prec. } \\
\text { pluvial } \\
(\mathrm{mm})\end{array}$ & casos & $\begin{array}{c}\text { Prec. pluvial } \\
(\mathrm{mm})\end{array}$ & casos & $\begin{array}{c}\text { Prec. pluvial } \\
(\mathrm{mm})\end{array}$ \\
\hline 2005 & 276 & 395,05 & - & - & - & - & 194 & 382,6 & 147 & 795,1 \\
\hline$* 2006$ & 158 & 406,05 & 222 & 362,1 & - & - & 310 & 405,85 & 472 & 245,6 \\
\hline 2007 & - & - & 246 & 425,8 & 516 & 284,45 & 454 & 506,25 & - & - \\
\hline$* * 2008$ & 273 & 458,4 & - & - & 302 & 422,05 & 263 & 577,75 & - & - \\
\hline
\end{tabular}

*2006 = octubre (314 casos/precipitación pluvial de 595,95mm)

**2008=la mayor incidencia se reportó en enero (311 casos/precipitación pluvial de $31 \mathrm{~mm})$ y octubre $(257$ casos/ precipitación pluvial de 615,95 $\mathrm{mm})$

Durante 2009, el comportamiento es similar: se encontró una alta incidencia en marzo (224 casos/precipitación pluvial de 34,35mm), setiembre (244 casos/ precipitación pluvial de 261,1mm), noviembre (227 casos/precipitación pluvial de 531,8mm) y diciembre (222 casos/precipitación pluvial de $86,85)$ 
En el 2010, la mayor incidencia se encuentra durante la estación lluviosa en junio (532 casos/precipitación pluvial de 409,1mm), setiembre (507 casos/precipitación pluvial de 680,1 $\mathrm{mm}$ ), octubre (471 casos/precipitación pluvial de 554,3mm), noviembre (366 casos/precipitación pluvial de 1156mm) y diciembre (432 casos/precipitación pluvial de 205,05mm).La incidencia anual de esta enfermedad tuvo el siguiente comportamiento:

\begin{tabular}{c|c}
\hline Año & No. de casos \\
\hline 2005 & 1827 \\
\hline 2006 & 2540 \\
\hline 2007 & 2691 \\
\hline 2008 & 3090 \\
\hline 2009 & 2077 \\
\hline$* 2010$ & 4137
\end{tabular}

*Presenta una precipitación pluvial superior al resto del periodo en estudio, lo cual coinciden con la mayor incidencia de este problema de salud.

7. Infecciones respiratorias agudas (IRAS): Respecto de esta enfermedad, se observó que durante el año 2005 la mayor incidencia se presentó durante la estación lluviosa específicamente en mayo (1566 casos/precipitación pluvial de 395,05 mm), junio (1779 casos/ precipitación pluvial de 331mm) y agosto(1800 casos/precipitación pluvial de 382,6mm). En el 2006, se reportó la mayor incidencia en marzo (2212 casos/precipitación pluvial de 54,95 mm), mayo (2254 casos/precipitación pluvial de 406,05 mm), agosto (2723 casos/precipitación pluvial de 405,85mm) y setiembre (1865 casos/precipitación pluvial de 245,6mm).En el 2007, hubo reportes en mayo (1215 casos/precipitación pluvial de 310,55mm), julio (2074 casos/ precipitación pluvial de 284,45mm), agosto (2113 casos/precipitación pluvial de 506,25mm) y octubre (1886 casos/precipitación pluvial de 698,95mm). En el 2008, se destaca enero (1819 casos/precipitación pluvial de $31 \mathrm{~mm}$ ), junio (2266 casos/precipitación pluvial de $325,5 \mathrm{~mm}$ ) y julio (2250 casos/precipitación pluvial de 422,05mm) con la mayor cantidad de casos reportados. El 2009 rescata los meses que presentaron mayor incidencia fueron: abril (1599 casos/precipitación pluvial de 130,25mm), junio (1448 casos/precipitación pluvial de 321,2mm) y julio (1590 casos/precipitación pluvial de 167,85mm); la misma situación se dio durante el 2010 en junio (4398 casos/precipitación pluvial de 409,1mm), setiembre (3266 casos/precipitación pluvial de 680,1mm), octubre (3339 casos/ precipitación pluvial de 554,3mm) y noviembre (3827 casos/precipitación pluvial de 1156mm)

Obsérvese el total anual de los casos presentados en el quinquenio:

\begin{tabular}{c|c}
\hline Año & No. de casos \\
\hline 2005 & 15921 \\
\hline 2006 & 20277 \\
\hline 2007 & 17964 \\
\hline 2008 & 20188 \\
\hline 2009 & 14147 \\
\hline 2010 & 35827 \\
\hline
\end{tabular}


8. Leishmaniasis: se encontró que esta enfermedad tiene una alta incidencia durante la estación lluviosa; al respecto, obsérvese los siguientes datos:

\begin{tabular}{l|c|c|c|c|c|c|c|c|c|c}
\hline \multirow{2}{*}{ Año } & \multicolumn{2}{|c|}{ Mayo } & \multicolumn{2}{c|}{ Julio } & \multicolumn{2}{c|}{ Agosto } & \multicolumn{2}{c|}{ Octubre } & \multicolumn{2}{c}{ Noviembre } \\
\cline { 2 - 10 } & casos & $\begin{array}{c}\text { Prec. } \\
\text { pluvial } \\
(\mathrm{mm})\end{array}$ & casos & $\begin{array}{c}\text { Prec. } \\
\text { pluvial } \\
(\mathrm{mm})\end{array}$ & casos & $\begin{array}{c}\text { Prec. } \\
\text { pluvial } \\
(\mathrm{mm})\end{array}$ & casos & $\begin{array}{c}\text { Prec. } \\
\text { pluvial } \\
(\mathrm{mm})\end{array}$ & casos & $\begin{array}{c}\text { Prec. } \\
\text { pluvial } \\
(\mathrm{mm})\end{array}$ \\
\hline 2005 & & - & 10 & 264,4 & 9 & 382,6 & & & 14 & 270,3 \\
\hline 2006 & 11 & 406,05 & - & - & - & - & 9 & 595,95 & - & - \\
\hline$* 2007$ & 9 & 310,55 & - & - & - & - & - & - & - & - \\
\hline 2008 & - & - & 3 & 422.05 & 4 & 577,75 & - & - & - & - \\
\hline
\end{tabular}

En el 2009 se da una situación interesante y es que la mayor cantidad de casos se da durante enero (6 casos/precipitación pluvial de 55,1mm) y abril (6 casos/precipitación pluvial de 130,25mm). Finalmente, durante el 2010, la mayor cantidad de casos reportados se da en junio (5 casos/precipitación pluvial de 409,1 mm), setiembre (4 casos/precipitación pluvial de 680,1 mm), octubre (11 casos/precipitación pluvial de $554,3 \mathrm{~mm}$ ), noviembre (6 casos/precipitación pluvial de $1156 \mathrm{~mm}$ ) y diciembre ( 8 casos/ precipitación pluvial de 205,05mm)

9. Leptospirosis: se encontró que durante el 2005 la mayor cantidad de casos reportados se dio en los meses de febrero (3 casos/precipitación pluvial de 27,35mm) y julio (2 casos/precipitación pluvial de 264,40mm) En el 2006, la mayor incidencia correspondió a agosto (2 casos/precipitación pluvial de 405,8mm). En el 2007, se presentó en 1 enero (3 casos/precipitación pluvial de 21,5mm), octubre (3 casos/precipitación pluvial de $698,95 \mathrm{~mm}$ ) y noviembre (3 casos/precipitación pluvial de 282,1mm)En el año 2008, entre los meses de mayor incidencia se encuentra marzo (3 casos/precipitación pluvial de 72,3 mm), junio (3 casos/precipitación pluvial de 325,5 mm), setiembre (5 casos/precipitación pluvial de 388,9 mm) y diciembre (3 casos/precipitación pluvial de 113,35mm), mientras que en el 2009, se dio en enero (9 casos/precipitación pluvial de 55,1mm), setiembre (5 casos/precipitación pluvial de $261,1 \mathrm{~mm}$ ), octubre (4 casos/precipitación pluvial de $365,55 \mathrm{~mm}$ ) y noviembre (4 casos/precipitación pluvial de $531,8 \mathrm{~mm}$ ). Finalmente, en el 2010 se reportó la mayor incidencia en junio (3 casos/precipitación pluvial de 409,1mm), julio (5 casos/ precipitación pluvial de 144,6mm), agosto (3 casos/precipitación pluvial de 393,4mm) y setiembre (4 casos/precipitación pluvial de 680,1mm)

10.Tosferina: se encontró que durante los años del 2005 y 2006 no se reportan casos de esta enfermedad. Para el 2007 la mayor cantidad de casos reportados se dan en junio (13 casos/precipitación pluvial de $425,8 \mathrm{~mm}$ ), julio (47casos/ precipitación pluvial de $284,45 \mathrm{~mm}$ ) y octubre (26 casos/precipitación 
pluvial de 698,95mm). En el 2008, la incidencia más alta ocurrió en agosto (31 casos/precipitación pluvial de $577,75 \mathrm{~mm}$ ), setiembre ( 89 casos/precipitación pluvial de 388,9mm), octubre (69 casos/precipitación pluvial de $615,95 \mathrm{~mm}$ ) y noviembre (41 casos/precipitación pluvial de $344,9 \mathrm{~mm}$ ). Durante el año 2009, la mayor incidencia se presentó en la estación seca, específicamente en enero (12 casos/precipitación pluvial de $55,1 \mathrm{~mm}$ ), febrero (14 casos/precipitación pluvial de $8,15 \mathrm{~mm}$ ) y marzo (12 casos/precipitación pluvial de $34,35 \mathrm{~mm})$. Finalmente, en el 2010 los meses en los que se reportaron la mayor cantidad de casos fueron marzo (5 casos/precipitación pluvial de 83,85mm), junio (3 casos/precipitación pluvial de 409,1mm), julio (3 casos/precipitación pluvial de 144,6mm) y noviembre (3casos/precipitación pluvial de 1156mm)

\section{DISCUSIÓN}

El aumento en la precipitación pluvial suele afectar la incidencia de algunas de las enfermedades de reporte obligatorio. Estas enfermedades por sus características e implicaciones sociales y económicas tienen especial importancia en el sistema nacional de salud, entidad vigilante de padecimientos que pueden expandirse rápidamente y afectar seriamente la salud de la población en general.

Este es el caso de los accidentes ofidicos que tienden a aumentar su incidencia con la precipitación, tal como lo refieren Blaikie et al. (1996) y Malilay (2000), quienes afirman que las inundaciones afectan el hábitat normal de las serpientes, las cuales se ven obligadas, debido al nivel del agua, a migrar a zonas secas donde usualmente están emplazados los seres humanos. Tal dato coincide con los resultados encontrados especialmente durante el año 2005, en el que al aumentar la precipitación, también se observa un aumento de los casos de accidentes ofídicos reportados.

No obstante, durante la estación seca, en algunos años se observó una incidencia alta, relacionada con la existencia de otros factores, como las quemas como preparación de los campos para el cultivo y los incendios forestales. Por otro lado, en el 2007, inicia una disminución en los accidentes ofídicos, lo cual puede estar relacionado con la expansión de las áreas urbanas, la deforestación y las quemas que también pueden estar incidiendo en los hábitats y los ciclos reproductivos de estos animales.

Es importante destacar que la frecuencia con que estos eventos se presentan depende de una gran cantidad de factores y de la cantidad de reptiles que existan en la zona. También, debe considerarse que se está trabajando con casos de accidente ofídicos, pero que no se tienen datos sobre la zona geográfica de donde proceden y si coinciden específicamente con el área donde se presenta la inundación.

La Asociación Colombiana de Sociedades Científicas \& Melgarejo (2010) mencionan que

el Ministerio de la Protección Social hizo pública su preocupación por la posibilidad de que, una vez cesen las lluvias, se registre un incremento sustancial de infecciones respiratorias, males 
intestinales, malaria, dengue, leptospirosis y mordeduras de serpientes. Además afirman que, las serpientes buscan los lugares secos, que son los mismos que buscan las personas para protegerse de las inundaciones. Esto desemboca, necesariamente, en un incremento de los accidentes ofídicos(s.f.)

Respecto del Dengue, en mayo, especialmente, aumentan los casos relacionados con la precipitación. Se debe tomar en cuenta que el comportamiento del vector trasmisor de esta enfermedad, cuyo huevo es capaz de sobrevivir la desecación hasta por un año, ya que en mayo inician las lluvias, ambiente propicio para el aumento en la incidencia.

El dengue es una enfermedad considerada como un problema de salud pública en el mundo. Según datos de la OMS, citados por Chávez (2010) "aproximadamente de 50 a 100millones de individuos son infectados cada año en el mundo, de los cuales entre 200 y 500,000 contraen dengue hemorrágico y el $5 \%$ fallece” (p.76); cifra de la que se destacan los niños y menores de 15 años, grupo más vulnerable.

Por otro lado, la World Health Organization (2005) refiere que las inundaciones pueden incrementar potencialmente la transmisión de enfermedades por vectores, ejemplo de ello es el dengue común y el hemorrágico, la malaria y la fiebre amarilla.

Dentro de los resultados se encuentra que existen meses durante la estación lluviosa en los que disminuye la precipitación; sin embargo, los casos de dengue se mantienen o aumentan, escenario que puede estar vinculado con el estancamiento del agua, los ciclos de vida del mosquito y otras características propias de la zona.

Un aspecto que también se debe considerar es que el periodo que tarda una persona desde la picadura hasta presentar los síntomas de la enfermedad (período de incubación) es de 3 a 14 días, dato que se corrobora en mayo del 2006 cuando aumenta la precipitación y los casos de dengue en el mismo mes y el mes siguiente; además, en mayo del 2008 aumentó también la precipitación y el mes siguiente incrementa significativamente la incidencia de la enfermedad.

La relevancia de esta enfermedad estriba en el costo de la atención de personas con dengue que, según Wong-McClure, R., Suárez-Pérez, X., Badilla-Vargas, X. (2007); en el 2002 ascendía a 465876441 colones, un desastre dentro del sistema nacional de salud; además de que son costos que difícilmente se sumen al impacto de las inundaciones en el país.

Por otro lado, no se debe perder de vista que si bien el aumento en la precipitación genera el ambiente propicio para la multiplicación del vector, el impacto de este aumento en número de insectos no se dará de forma inmediata, mas sí afectará a las personas que perdieron las casas o que vieron afectada su capacidad adquisitiva por efecto directo del de las lluvias intensas o de las inundaciones. 
En la misma línea, el tiempo de incubación del insecto, así como el periodo transcurrido desde que la persona es picada hasta que desarrolla la enfermedad, afecta seriamente la vinculación de los casos con el comportamiento de la precipitación, ya que pueden transcurrir semanas, e incluso meses, desde que se dio el evento hasta que se presenta el brote, lo que en parte justifica el comportamiento de esta enfermedad.

Se destaca que el sistema de salud de Costa Rica implementa una serie de estrategias dirigidas a atenuar el impacto de esta enfermedad, tal es el caso de las campañas televisivas sobre la importancia de eliminar criaderos, las campañas de fumigación entre otras. Todo esto sin perder de vista la labor de diferentes profesionales de salud como las enfermeras (os) que realizan barridos epidemiológicos y otras estrategias con la finalidad de debilitar los brotes de esta enfermedad.

Todo lo anterior permite afirmar que sí existe una relación significativa entre el aumento en la precipitación y el comportamiento del dengue en Pérez Zeledón, mediado por el impacto que tiene la estación lluviosa en el ambiente, así como el ciclo de vida del insecto y el periodo de incubación de la enfermedad en las personas.

El aumento de la precipitación puede estar relacionado con otros problemas de salud, tal es el caso de las enfermedades diarreicas, las cuales tienen una multicausalidad, sin olvidar que la contaminación del agua, el hacinamiento y otras situaciones que suelen presentarse con el aumento en la precipitación tienden a tener un peso importante en su incidencia.

Esta problemática ha sido ampliamente divulgada por los medios de comunicación, ejemplo de ello es el artículo publicado en el Diario Al Día de Costa Rica con la siguiente noticia:

La mala manipulación de alimentos y la no aplicación de medidas higiénicas dispararon los casos de diarrea en Parrita luego de las inundaciones. Edgar Carrillo, director del Área de Salud de Parrita, indicó que los casos pasaron de entre 10 y 12 semanales, a 60 que se contabilizaron la semana anterior. (López, 2010)

Además, la relación entre las diarreas y los factores del clima se pueden establecer en dos sentidos.

Primero, por los desequilibrios hídricos y térmicos que causan períodos de sequías o inundaciones y que alteran el ambiente de desarrollo de bacterias y virus. Segundo, la contaminación de fuentes de aguas (almacenadas y tomas de agua para uso poblacional) o la descomposición de alimentos perecederos durante eventos climatológicos extremos y cuya ingestión causa problemas gastrointestinales (Instituto Meteorológico Nacional \& Ministerio de Salud, 2008, s.p.) 
Los párrafos anteriores afirman la relación existente entre las inundaciones y los casos de diarrea, información respaldada por Blaikie et al. (1996), al afirmar que las inundaciones traen riesgos de enfermedades como el cólera y la disentería, quepodría originarse en las aguas negras esparcidas y la contaminación del agua potable.

En cuanto al impacto económico de este problema de salud, el costo promedio de atención por diarreas para el período 1997-2006 es de 9 millones de dólares (casi 5000 millones de colones al tipo de cambio actual), cifra que representa cerca del 0,2\% del PIB (Instituto Meteorológico Nacional \& Ministerio de Salud, 2008).

Se destaca que existen dos bacterias que suelen causar una patogenia más severa en individuos susceptibles, tal es el caso de la Shigellosis y la Salmonelosis. Su virulencia ha llevado a separar las diarreas causadas por ellas del conglomerado "diarreas".

En relación con la primera, Mejía (2007) afirma que

La ruta fecal-oral es la forma principal de transmisión, a través de la ingestión de alimentos o agua contaminada, siendo los niños de 1 a 4 años de edad los más afectados. Los síntomas se presentan en forma abrupta después de 2 a 4 días de incubación, con presencia de fiebre elevada, toxicidad, anorexia, náusea, vómitos, calambres abdominales y diarrea. Inicialmente, la diarrea implica la aparición de deposiciones líquidas abundantes (afectación de intestino delgado), seguido de deposiciones muco-sanguinolentas frecuentes de pequeño volumen, asociada a pujo y tenesmo (compromiso de intestino grueso). Más del 50\% de casos es posible que no progresen a una disentería. (p. 80)

Por otro lado, de acuerdo con Noji (2000), después de los eventos de inundación surge el temor dentro de las autoridades de salud pública por los brotes de enfermedades transmisibles. Aquí se menciona que existe un potencial riesgo para la transmisión de enfermedades transmitidas por el agua, de las que la shigela es un claro ejemplo.

Además de lo mencionado, es necesario agregar que el desborde de tanques sépticos, contaminación de pozos de agua, así como los malos hábitos higiénicos al momento de preparar los alimentos, son detonantes de este tipo de enfermedad.

Sin embargo, se destaca que para el periodo en estudio y la zona existen muy pocos registros, lo cual puede verse relacionado con la falta de especificidad en el diagnóstico, de allí que sea tratada como un problema diarreico más, tanto a nivel hospitalario como doméstico, razón que obliga a aclarar que su inclusión se debe a que por su modo de transmisión suele estar muy vinculado al aumento en la precipitación. 
En cuanto a la salmonellosis, esta se trata de una enfermedad bacteriana que, según Benenson (1997)

se manifiesta por enterocolitis aguda, de comienzo repentino, que incluye cefalalgia, dolor abdominal, diarrea, náuseas y a veces vómito, además el periodo de incubación de esta enfermedad va de 6 a 72 horas posterior a la ingesta de agua o alimentos contaminados (p. 402)

No se debe perder de vista que se trata de una diarrea generadapor una bacteria muy patógena. Sin embargo, dependiendo de la vulnerabilidad de la persona infectada, puede tratarse a nivel doméstico o no realizarse los exámenes pertinentes para identificarla como salmonelosis, lo cual podría llevar a su subregistro.

Al igual que con la shigela, existe poca información para poder establecer una relación entre el aumento en la precipitación pluvial y la incidencia de esta enfermedad. Se requiere más datos y un seguimiento puntual de las áreas que se ven más afectadas por el aumento en la precipitación, pues así se sabe si los casos reportados corresponden a estas áreas, además de que permite filtrar el subregistro.

Por otro lado, los problemas virales respiratorios se presentan con mucha frecuencia durante la estación lluviosa; en su mayoría;son agrupados como influenza e IRAS (infecciones agudas de las vías aéreas superiores), de lo cual es importante mencionar que con mucha frecuencia son tratadas en el hogar y que algunas veces la influenza es vista como una IRAS ya que la sintomatología es muy similar.

En relación con la influenza y su comportamiento se sabe que

se trata de una enfermedad virica de las vías respiratorias que se caracteriza por fiebre, cefalalgia(dolor de cabeza), mialgia(dolor muscular), postración, coriza(congestión y secreción nasal), dolor de garganta y tos. Además, presenta un periodo de incubación relativamente corto de uno a tres días y que se transmite de manera muy simple ya que se encuentra en el ambiente o en la saliva o secreciones de las personas infectadas (Benenson, 1997, p.267)

Es significativo recordar que debido al traslado de personas a un albergue, la humedad y el hacinamiento permiten la diseminación de la enfermedad, cuadro que aumenta en estos periodos. Además, vale la pena recordar que esta enfermedad tiene un comportamiento estacional y que muchas personas contraen el virus, pero no desarrollan la enfermedad.

Además, es importante tomar en cuenta que la influenza es un problema de salud que tiene un comportamiento estacional; sin embargo, por su modo de contagio, el aumento en las precipitaciones pluviales se convierten en un ambiente propicio para la transmisión de estos virus.

Respecto de las IRAS, el otro problema respiratorio, se sabe que bajo este título se agrupan innumerables enfermedades respiratorias agudas de origen vírico identificado o supuesto, su forma de 
contagio se da por contacto directo o por inhalación de gotitas llevadas por el aire; indirectamente, también se puede dar el contagio por medio de las manos y los artículos recién contaminados con exudados nasofaríngeos de personas enfermas (Benenson, 1997) en cuanto al periodo de incubación,

"una persona puede tardar de 12 horas a 5 días en presentar la infección, mas sin embargo es importante destacar que en los países tropicales su incidencia aumenta durante la época lluviosa y en los templados durante el otoño y la primavera."(Benenson, 1997, p.494)

Dicho escenario es muy evidente para el periodo estudiado, ya que se aprecia cómo a partir de mayo se presentó un aumento en la incidencia de IRAS, con presencia de algunos repuntes relacionados con el aumento en las lluvias.

El aumento en la precipitación también puede afectar los ciclos de vida de otros vectores y por consiguiente la incidencia de la enfermedad que trasmite lo transmite, ejemplo de ello es la Leishmaniasis "una infección de la piel causada por especies del género Leishmania, se considera una zoonosis transmitida, en América, por la picadura del mosquito Lutzomyia; además se da frecuentemente en zonas húmedas, selváticas, tropicales y subtropicales, durante estaciones lluviosas” ( Sánchez, Mattar, González, 2009, p. 98)

Cabe mencionar que esta enfermedad es parasitaria, se presenta en la piel y membranas mucosas y es transmitida a las personas por un vector flebótomo hembra (tipo de mosca) a las personas, de ahí que los ambientes húmedos posteriores al inicio de la estación lluviosa y a los eventos de inundación se convierten en espacios propicios para la multiplicación del vector. Lo anterior, se corroboró a partir de los datos que muestra un aumento en la incidencia de esta enfermedad a partir de mayo con el aumento en la precipitación. Otro aspecto importante es que

el protozoario requiere de un período de incubación de 2 a 6 semanas que inicia con la picadura por el flebótomo, provocando una lesión que generalmente es una pápula eritematosa con una vesícula en su vértice, la cual crece hasta formar una bula o una pústula que a los pocos días se ulcera, deja una superficie roja granulosa con bordes duros ligeramente levantados(Sánchez et al., 2009, p. 98)

Lo anterior, se debe tomar en cuenta al realizar el análisis y al vincularlo con el aumento en la precipitación; además, sirve como guía para el personal de salud a la hora de implementar campañas para controlar el vector y disminuir la incidencia de esta enfermedad.

Otro problema de salud que se relaciona con los cambios ambientales generados por el aumneto en la precipitación es la leptospirosis, la cual es transmitida a través de la orina de animales portadores o el contacto con material contaminado.

Benenson (1997) afirma que existen problemas para el diagnóstico, ya que 
la leptospirosis a veces se diagnostica erróneamente como meningitis, encefalitis o influenza, y las pruebas serológicas de la infección leptospirósica se advierten en $10 \%$ de los casos con meningitis y encefalitis, por lo demás no diagnosticada.... La enfermedad clínica dura de unos pocos días a tres semanas o mas. En términos generales muestra dos fases: la leptospirémica o febril, seguida de la convalecencia o fase inmune.... El restablecimiento de los casos no tratados puede durar varios meses. Las infecciones pueden ser asintomáticas. (p.294)

Retomando lo que afirma Benenson, en Costa Rica podría existir un subregistro de casos, ya que estarían mal diagnosticados o, inclusive, podrían estar manejandose en los hogares como resfríos y no llegar a solicitar atención médica.

También es importante mencionar que dependiendo de la susceptibilidad del huésped, puede tratarse de una enfermedad aguda y generalizada que se caracteriza por una vasculitis infecciosa que provoca innumerables alteraciones en órganos nobles como pulmones, hígado y riñones, por ello "afecta al ser humano y a los animales en forma endémica, representando un problema de salud pública en los países latinoamericanos" (Ávila, Escalona y Rodríguez, 2010, s.p.)

El que sea un problema de salud pública, y su vínculo con el aumento en la precipitación, se asocia también a que, cuando aumenta la precipitación, con mucha frecuencia las personas se ven expuestas a agua y lodo que arrastra materia contaminada con excremento de animales infectados. En este sentido

Se calcula que un $25 \%$ de los roedores y entre el 40-60\% de los perros de áreas urbanas, están colonizados o presentan anticuerpos específicos, transformándose de esta forma en portadores asintomáticos. La forma de transmisión es a través del agua, barro, basura u otros elementos contaminados con orina (Del Pont \& Laube, 2001, p. 75)

De esta manera, se comprende por qué el aumento en la precipitación facilita el contagio de esta enfermedad, ya que según Del Pont y Laube (2001), la forma en que ingresa al individuo es por piel o mucosas; además, los autores señalan que la ingestión de agua contaminada no representa un riesgo de contagio.

Es importante destacar la relación entre el aumento en la precipitación y dicho problema de salud, ya que según Sánchez et al. (2009), ciertas enfermedades transmitidas por roedores, como la leptospirosis, la tularemia y las virosis hemorrágicas, se asocian con las inundaciones.

En Costa Rica, cuando se inicia la temporada lluviosa se da un aumento en la precipitación, lo cual genera eventos de inundación en algunas ocasiones. Usualmente, se desbordan tanques sépticos y todo tipo de aguas negras; además, el agua arrastra lodo y otros materiales (heces y orina de animales en gran cantidad) a lo largo de las zonas afectadas, por lo cual puede existir una relación entre el aumento en la precipitación pluvial y la incidencia de los casos de esta enfermedad. 
Por último, la tosferina, un problema de salud reemergentecuyo comportamiento se vincula poco con las precipitaciones, se trata de "...una enfermedad bacteriana altamente contagiosa, que ocasiona una tos violenta e incontrolable y que puede dificultar la respiración” (Benenson, 1997, p.447).

En Costa Rica existe un programa de vacunación para esta enfermedad; sin embargo, Riffelmann, M., Littmann, M., Hellenbrand, W., Hülbe, C., Heinz, C (2008), en su revisión sistemática, afirman que el efecto de protección inducida por las vacunas es de aproximadamente $85 \%$ después de tres dosis y tiene una duración de al menos cinco años, tras lo cual se observa una disminución gradual en la inmunidad.

Esta situación, según los autores mencionados, justifica los casos que se presentan en escolares, adolescentes y adultos. Otro dato importante es que uno de los grupos más vulnerables son los mayores de 60 años, quienes presentan aproximadamente el $40 \%$ de las complicaciones asociadas a la enfermedad.

Por otro lado, a pesar de que por muchos años se consideró una enfermedad de niños y lactantes, en las últimas décadas, esta situación ha venido cambiando. De acuerdo con Ávila-Agüero (2007)

desde la década de los 80 se ha identificado a nivel mundial un cambio epidemiológico caracterizado por un aumento en el reporte de casos y con brotes cada 3-4 años, afectando adolescentes y adultos que se convirtieron en la principal fuente de contagio de lactantes(s.p)

En síntesis, el conocimiento que se pueda ir acumulando sobre estas enfermedades y el mejor registro de los casos es fundamental para disminuir el impacto que estos eventos tienen sobre las comunidades afectadas.

\section{CONCLUSIONES}

- $\quad$ El aumento en la precipitación constituye un detonante para el aumento en la incidencia de muchos problemas de salud, lo cual, según la vigilancia epidemiológica, es una acción fundamental de las instancias de salud para gestionar el riesgo de estas enfermedades.

- Los registros más detallados permiten una mejor vinculación entre las personas enfermas y las áreas afectadas por el aumento en la precipitación pluvial.

- $\quad$ El impacto económico en la atención de estos problemas de salud y la necesidad de sumarlo al costo de la atención de las emergencias asociadas a precipitación confiere mayor peso a la gestión del riesgo.

- $\quad$ El aumento en la precipitación tiene efectos sobre el medio ambiente que repercuten en la salud de las comunidades afectadas, por tanto, la educación y las intervenciones que realizan los y las profesionales de enfermería son pieza fundamental para mitigar el impacto que esto genera. 
- $\quad$ Existen problemas de salud que tienen una causalidad múltiple, por lo que se requiere investigaciones más profundas, seguimiento de las personas y las áreas afectadas, así como la mejora en los sistemas de registro, de manera que permitan el establecimiento de relaciones entre ambos fenómenos.

\section{REFERENCIAS}

Ahern, M., Kovats, R., Wilkinson, P., Few, R. \& Matthies, F.(2005). Global Health Impacts of Floods: Epidemiologic Evidence. Oxford Journals. 27 (1) 36-46

Asociación Colombiana de Sociedades Científicas \& Melgarejo, S.(2010). Tras las inundaciones vendrían las epidemias. Recuperado de http://www.eltiempo.com/vida-de-hoy/salud/tras-las-inundaciones-vendrianlas-epidemias_8615380-4

Avila-Agüero, M.(2007). Tosferina: infección reemergente. Acta médica costarricense, 49(3) p. 134-135

ÁVILA, J., ESCALONA, R. y Rodríguez, A.(2010). Método Práctico para el control de leptospirosis. Recuperado de http://www.cocmed.sld.cu/no142/pdf/no142ori10.pdf

Benenson, A. (1997) Manual para el control de las enfermedades transmisibles. Washington, D.C., Estados Unidos de América: Ed. OPS. 569.

Blaikie, P., Cannon, T., Davis, I. y Wisner, B.(1996). Vulnerabilidad: El entorno social, político y económico de los desastres. Ciudad de Panamá, Panamá: Ed. LA RED. 290.

Chávez, M. (2010). Después de las inundaciones: el dengue.Recuperado de: http://web.ebscohost.com/ehost/pdfviewer/pdfviewer?sid=4aea608d-3671-4106-8519

ebe4c4584c67\%40sessionmgr110\&vid=1\&hid=111

Del Pont, J., Loube, G.(2001). Leptospirosis: una realidad. Arch. Argent. Pediatr, 99(1): 75-76

DesInventar (2011).Costa Rica: Inventario Histórico de Desastres. Recuperado de:http://online.desinventar.org/

DesInventar (2011). Sistema de Inventario de efectos de los desastres. Recuperado de: http://online.desinventar.org/?lang=spa

Fallas, J., Valverde, C.(2007). Inundaciones en Costa Rica y estudio de caso en una cuenca forestada del Caribe Costarricense: Evidencia de los últimos 34 años. Recuperado de:http://www.mapealo.com/Costaricageodigital/Documentos/noticias/ponencias/Bosques\%20e\%20inundaci ones\%20en\%201a\%20Cuenca\%20del\%20rio\%20Matina\%20nov2007.pdf. 
Fariñas, A., Coutin, G., Rodríguez, D. (2009). Una reflexión histórica sobre la vigilancia en salud en Cuba. Revista Cubana de Salud Pública. 35(2)

Fewtrell, L. \& Kay, D. (2008). An attempt to quantify the health impacts of flooding in the UK using an urban case study. Public Health. Recuperado de:www.elsevierhealth.com/journals/pubh

Flores, R., Salas, J., Astorga, M., Rivera, J. (2010). El impacto económico de los eventos naturales y antrópicos extremos Costa Rica, 1998-2009.Recuperado de: http://www.crid.or.cr/digitalizacion/pdf/spa/doc18108/doc18108-contenido.pdf

Giorgadze, T., Maisuradze, I., Japaridze, A., Utiashvili, Z. ,Abesadze, G.(2011) .Disasters and their consequences for public health. Georgia Med News. Recuperado de: http://www.ncbi.nlm.nih.gov/pubmed/21685525\#

Instituto Nacional de Estadística y Censo (2010). Cifras preliminares de población y vivienda del censo 2011. Recuperado de: http://www.inec.go.cr/Web/Home/Noticia.aspx?id=2

Instituto Costarricense de Electricidad (ICE) (2011). Precipitación pluvial estaciones meteorológicas Linda y Alto San Juan. San José. Costa Rica

Instituto Meteorológico Nacional y Ministerio de Salud (2008). Efectos del Clima, su variabilidad y cambio climático sobre la salud humana en Costa Rica. Recuperado de: http://cglobal.imn.ac.cr/Pdf/adaptacion/SALUD-CLIMA.pdf

López, A (19 de noviembre del 2010). Crecen los casos de diarrea. Al Día. Recuperado de: http://www.aldia.cr/ad_ee/2010/noviembre/19/nacionales2594905.html

Malilay, J. (2000).Inundaciones: Antecedentes y naturaleza de las inundaciones. Recuperado de: http://www.greenstone.org/greenstone3/nzdl;jsessionid=CAF953E0248C1C3752A191406FCD414D?a=d\&d $=$ HASHa5f1acedd6b06571b517c7.9.pp \&c=helid\&sib=1\&dt=\&ec=\&et=\&p.a=b\&p.s=ClassifierBrowse\&p.sa Mejía, H. (2007). Opciones de tratamiento en shigelosis. Rev. Soc. Bol. ped., 46(1) 80-84. Recuperado de http://www.scielo.org.bo/pdf/rbp/v46n1/v46n1a14.pdf

Municipalidad de Pérez Zeledón (2011). Página principal. Recuperado de: http://www.mpz.go.cr/ NOJI, E.(2000). Impacto de los desastres en la Salud Pública. Bogotá, Colombia: Ed. OPS. 461

Riffelmann, M., Littmann, M., Hellenbrand, W., Hülbe, C., Heinz, C.(2008). Pertussis: not only a disease of childhood. Publicación Médica., 105 (37) 623-628 DOI: 10.3238/arztbl.20080623 


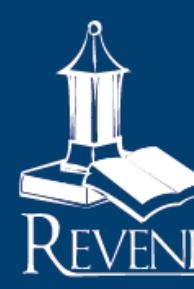

Revista Electrónica Enfermeria Actual en costa Rica

Sánchez, L., Mattar, S., González, M.(2009). Cambios climáticos y enfermedades infecciosas: nuevos retos epidemiológicos. Rev. MVZ Córdoba, 14 (3) 1876-1885

Wong-McClure, R., Suárez-Pérez, X., Badilla-Vargas, X. (2007). Estudio de la estacionalidad del dengue en la costa pacífica de Costa Rica (1999-2004). Acta médica. costarricense,49(1): 38-41

World Health Organization (2005). Weekly Epidemiological Record: Flooding and communicable diseases fact sheet. $W H O, 80(3):$ 21-28. 